\title{
Urethral stenosis of women about a case
}

\author{
Walid bai *, Mahdi Graiouid, Messian gallouo, larache yassine, doumer abderrahman, Jandou issam, Dakir Mohammed, Adil Debbagh, \\ Rachid Aboutaieb \\ University Hospital Center Ibn Rochd, Casablanca, Morroco \\ *Corresponding author: Walid bai, University Hospital Center Ibn Rochd, Casablanca, Morroco. Email: walid2724@gmail.com \\ Received date: December 07, 2019; Accepted date: December 16, 2019; Published date: December 19, 2019 \\ Citation: Walid B., Mahdi G., Messian G., larache Y., Doumer A., Jandou I., Dakir M., Adil D, Rachid A., Urethral stenosis of women about a \\ case, Clinical Medical Reviews and Reports, 1(1); DOI: 10.31579/CMRR/2019/002
}

Copyright: (C) 2019 Walid bai. This is an open access article distributed under the Creative Commons Attribution License, which permits unrestricted use, distribution, and reproduction in any medium, provided the original work is properly cited.

Abstract
Introduction: Non-tumoral urethral strictures are a rare clinical entity in women with little literature.
Observation: We report a case of a 56-year-old woman who consults for acute retention of urine with impossibility
of sounding, preceded by dysuria. She had an unexplained retention episode 8 years ago without urologic
investigations.
Conclusion: Urethral stricture is an infrequent condition in women, the most dangerous etiology being stenosis of
cancerous origin. Non-malignant tumoral causes such as that reported in our observation are exceptional.
Keywords: Stenosis; urethra; woman, urethroplasty

\section{Introduction:}

Urethral stricture in women is a rare clinical entity. It represents 3 to $8 \%$ of the obstructive causes of the low device in the woman [1]. Its incidence is significantly lower than in men. This may be explained by a shorter length than the male urethra, greater mobility and the fact that it is better protected by pelvic bones [2]. The search for a tumor should be done first by a biopsy, because it is the most feared etiology. Non-tumoral causes occur in 25 to $40 \%$ of urethral strictures. The etiology of non-tumor lesions is mainly obstetric trauma and anterior urogenital surgery, ie incontinent surgery, urethral diverticulum, vaginal gynecology surgery, and rarely endoscopic surgery. Radiation therapy, postmenopausal hormone deficiency vulvar atrophy, and acute and subacute urethritis are other possible causes of narrowing of the urethra in women. Periurethral fibrosis is the possible evolutionary complication whatever the etiology.

\section{Observation:}

Mrs. K.H aged 56, consults for acute retention of urine with impossibility of sounding. During the interview, the patient reports the notion of acute urinary retention that appeared in 2011, without fever, urethral burns or trauma in the antecedents. No urological investigation was performed at this time, it only benefited from a bladder catheterization and the catheter removed 10 days later; the survey was considered easy and not traumatic. She had no difficulty urinating in the process. Moreover, it does not present other medical or surgical pathologies. She is single and still a virgin.

Clinical examination reveals atrophy of the vaginal lips, a malformation of the vaginal opening and a rounded blistering lesion of the urethral meatus which occupies all the caliber of the meatus with impossibility of sounding. The vaginal touch not done, the patient being virgin (Fig. 1).

Magnetic resonance imaging (MRI), performed in August 2018, revealed a regular and significant non-tumoral thickening of the urethral walls over $20 \mathrm{~mm}$, its walls are in T1 isosignal and T2 hypersignal with regular thickening. bladder walls and a collapsed vaginal cavity (Fig. 2).

Uretro-cystography (UCR) shows narrowing of the distal urethra associated with a urethro-vaginal fistula (Figure 3).

A biopsy was performed. Histology concludes with a nonspecific inflammatory reaction with collagen fibrosis without signs of malignancy. It is proposed to perform a fistula treatment with progressive urethral dilatation successfully performed.

After discussion with the patient and her sensitization on the fact of coming consulted at the slightest finding of a decrease of the urinary flow for dilations, and that in case of a recurrence urethroplasty will be proposed. Since then, the patient is easily urinating and satisfied.

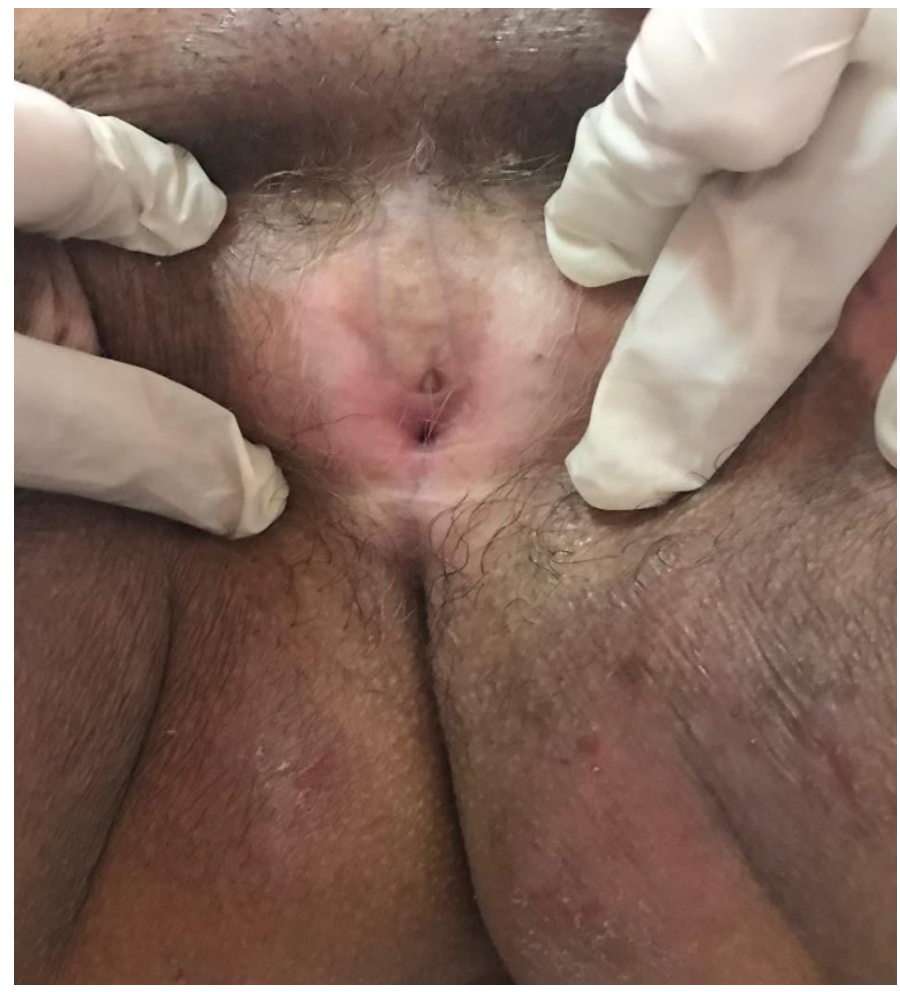

Figure 1: Aspect of the urethral meatus 


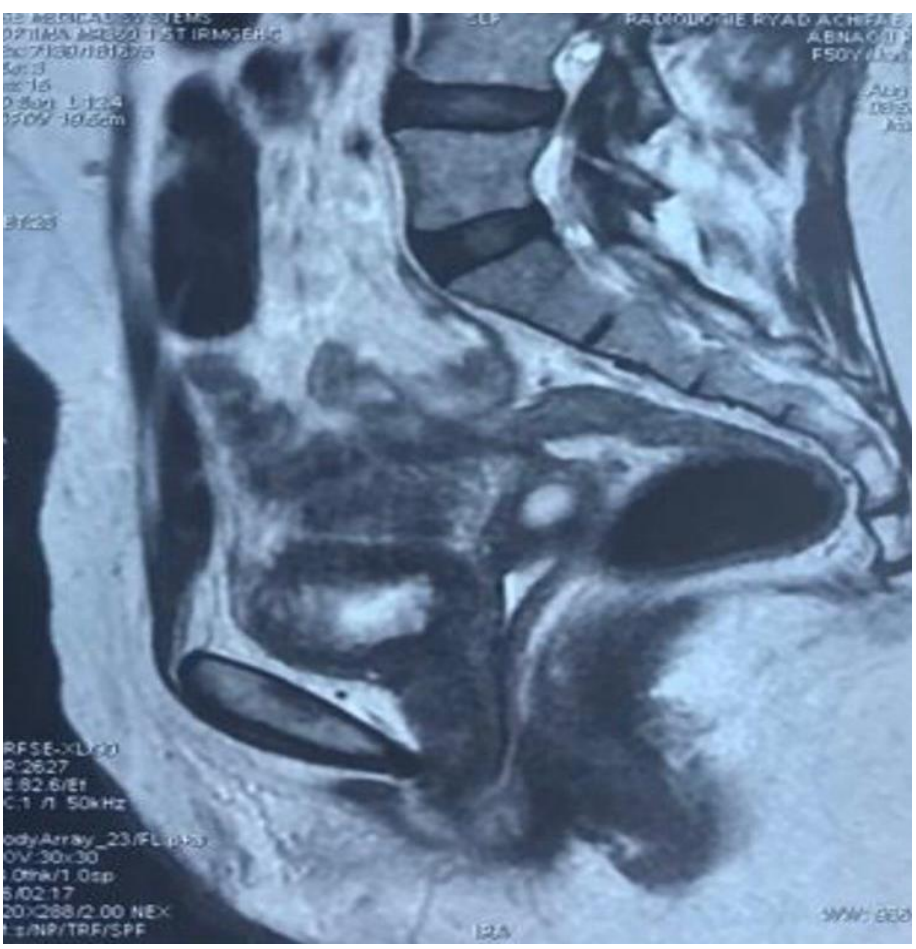

Figure 2: T2 sagittal section showing a thickening of the wall of the bladder and the urethra.

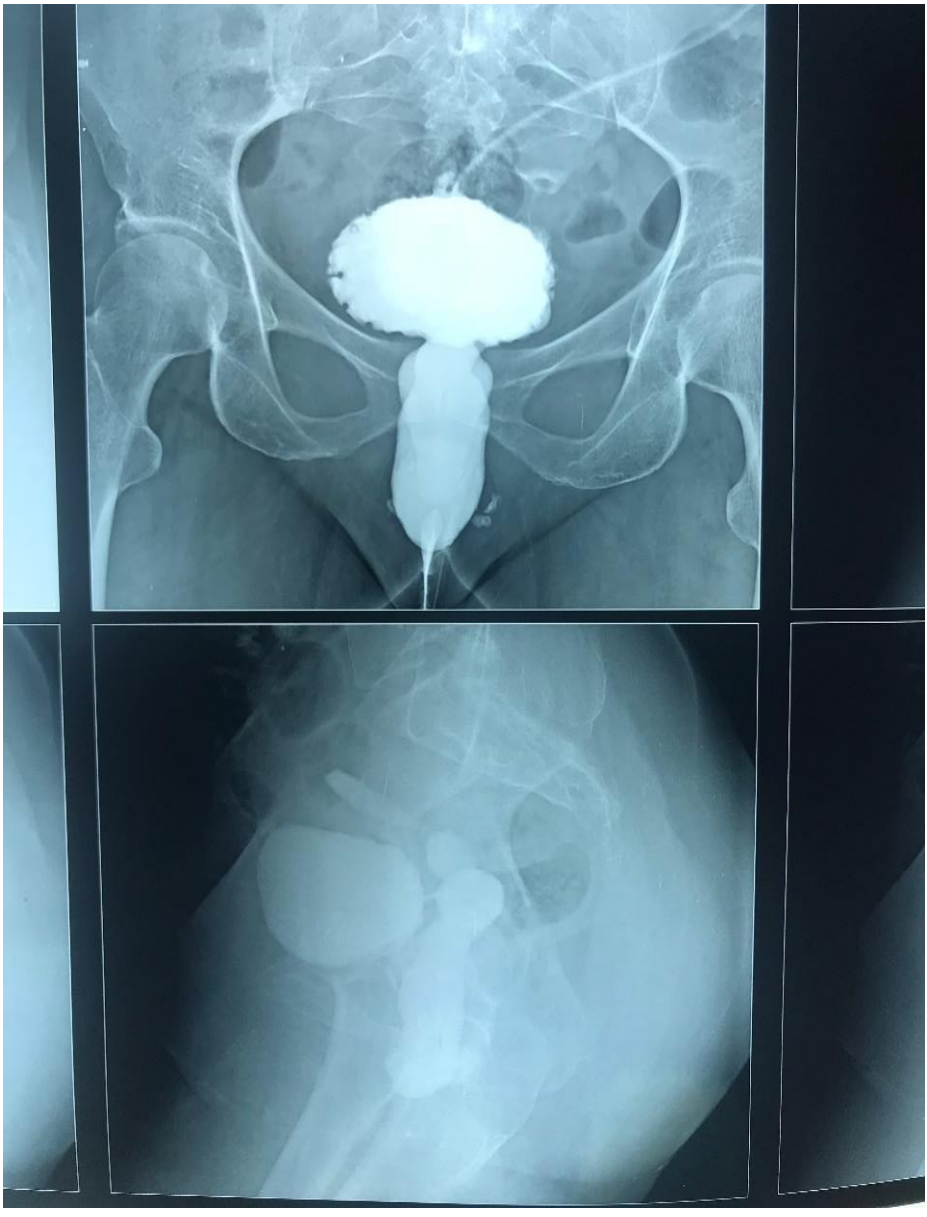

Fig 3: Uretro-cystography (UCR) shows narrowing of the distal urethra associated with a urethro-vaginal fistula

\section{Discussion:}

SUF was described by Brannan in 1951 and reported for the first time in 1828 [3]. The term urethral stricture refers to the healing of the urethral epithelium with or without spongiofibrosis. The result is a narrowing of the urethral light. Smith et al. [4] defined SUF as "a fixed anatomic narrowing of the urethra so that light cannot receive instruments without disturbance of the urethral mucosa".

Pugh [2] in 1924 conducted a study based on several clinical observations and the work of Lisfranc. In his study, it appears that urethral strictures are often of distal and meatal seat. The main etiologies he finds are gonococcal urethritis and obstetric trauma. The diagnosis was made by progressive calibration with probes or candles performing both a technical and therapeutic test.

\section{Symptom:}

Women with ED usually have lower urinary tract disorders such as low urine output, pollakiuria, urgency, and incontinence. Recurrent urinary tract infections can occur, and on rare occasions, urine retention [5].

\section{Epidemiology:}

The true incidence of SU is not known. Bladder obstruction in women is rare, and has been reported at less than $8 \%$ [6]. In a study of women with urodynamic signs and symptoms of urinary tract urology, SU accounted for only $7 \%$ of cases [7].

\section{Diagnosis:}

Urethral stenosis in women is defined as anatomical narrowing of the urethral caliber by urethral wall injury that does not permit or make difficult the catheterization of the urethra [8]. It must be distinguished from obstructive functional disorders that can be used in physiological and radiological investigations, but which are usually passable by a catheter. Contrary to man, the contracture of the sphincter, in case of vesicosphincter dyssynergia, is easily probed.

Careful examination of the patient should be done to eliminate extrinsic causes such as invasion of cervical cancer or vulva. It is also necessary to look for a hormonal deficiency with alteration of the trophicity and vaginal dryness.

The anamnesis will look for obstructive signs such as dysuria but also signs of overactive bladder by bladder control upstream of the obstacle.

Regarding the diameter from which to consider the urethra as stenosis, there is no consensus. Brannan [9] suggests that an urethra that does not allow the passage of a ch 20 probe is stenotic.

In the literature, obstructive disorders appear as soon as the urethra no longer allows the passage of a ch14 probe. The positive diagnosis of urethral stenosis in women is also based on imaging. Retrograde urethrocystography is a simple examination that reveals the stenosis, its extent, except in case of tight stenosis that cannot be catheterizable like our patient. In this case, vaginal ultrasound can be used and, especially, pelvic MRI which has the advantage of specifying the alterations of the urethral wall and looking for periurethral lesions.

\section{Etiology:}

The etiology of narrowing of the urethra in women in most cases is unknown. In developing countries the most common cause is urinary tract injury during prolonged labor during delivery.

In industrialized countries, the most frequent causes are: post-traumatic, iatrogenic injuries (during urethral diverticulectomies, endoscopic and gynecological surgeries), pelvic radiotherapy or acute and chronic urethritis [10]. Vulvar atrophy is another associated factor in postmenopausal women [10].

Edwards et al. [11] described on an animal model the effects of different 
materials and diameter of the probes on the urethral mucosa. They find that all materials cause inflammation of the mucosa. However, silicone and small caliber catheters are those that induce less inflammation. This can be explained by the fact that they allow the flow and evacuation of urethral secretions. Prolonged probing time increases the risk of bacterial colonization that promotes inflammation of the mucosa and increases the risk of stenosis. A probe left for a long time in traction on the urethra causes ischemia and devitalization of the urethral mucosa. The authors therefore recommend the use of small caliber silicone catheters placed in a non-traumatic manner and with the shortest possible sounding time. Proximal fixation of the probe should be done to prevent traction.

\section{Therapeutic management of urethral stricture in women:}

The goal of treatment of urethral stenosis is to correct anatomic and functional obstruction, with the preservation of urinary continence. The treatment of urethral strictures in women depends on the location and length of narrowing, the length of the proximal healthy urethra, the integrity of the bladder neck, and the existence of incontinence. [12]

\section{Dilation of the urethra}

Historically, dilatation of the urethra has been a pillar of ED treatment, although evidence of its effectiveness is limited. Some favorable longterm results for dilatation of the urethra for SU have been reported. Smith et al. reported a success rate of $57 \%$ at an average follow-up of 21 months in seven women after dilatation to $30 \mathrm{Fr}$. In the largest series published on urethral dilatation, Romman et al. had a success rate of $51 \%$ in 91 patients for initial dilatation to $41 \mathrm{Fr}$.

Endoscopic management with cold blade or laser incision of the midurethral stenosis was suggested, usually by incision at the $3 \mathrm{~h}$ and $9 \mathrm{~h}$ positions. The urethral catheter is recommended in these cases, with or without postoperative CIC. Urethrotomy associated with dilatation of the urethra showed short-term success in a single study involving ten patients. It is thought, however, that this treatment has a potential risk for the sphincter mechanism and may lead to urinary incontinence [12].

\section{Urethral reconstruction}

When urethral stenosis involves the distal urethral meatus in isolation, a meatotomy or a meatoplasty may be a prudent option. Circumferential excision of the stenosis of the meatus followed by approximation of the lumen of the urethra to the vaginal epithelium performed on a Foley probe has been described. Due to the more proximal continence mechanism in women, this may be appropriate for stenoses involving up to $1 \mathrm{~cm}$ of the distal urethra. [12]

Several reconstruction techniques for managing more extensive FUS have been described to date. These included vaginal or labial flaps, as well as vaginal and oral mucosa transplants.

\section{Vaginal flaps}

The vaginal flap urethroplasty includes some of the oldest published urethral reconstruction techniques. Blavais described it as an alternative to bladder neo-urethra formation in 1989. In 2002, Tanello et al. described their pedicle flap of the labia minora, which is a tunnel under the vaginal epithelial layer and which was successful in both patients with a 2-year follow-up. [12]

Montorsi et al. published the largest series of vaginal flap urethoplasty in 17 patients. Importantly, they represented narrowing of the distal urethra without prior intervention and used a dorsal approach. An $88 \%$ success was noted with a postoperative calibration of 28 Fr. Several published series examined the use of urethroplasty of the ventral vaginal flap with success rates between 80 and $100 \%$ [12]

\section{Vaginal grafts}

Uretroplasty with vaginal transplant was described by Tsivian and Sidi in 2006, two patients who had a successful reconstruction with a dorsal vaginal transplant. Petrou et al. Published the largest series of uterine and vaginal graft uretroplasties, based on the technique of Tsivian and Sidi. They reported their experience in 11 patients, three of them requiring a subsequent dilatation of the urethra during a followed by $6-46$ months. [12]

Three series examined the use of labial uterine graft uretroplasty in the ventral insert, with success rates ranging from 75 to $100 \%$, and average follow-up between 15 and 24 months.

Vaginal flaps and graft uretroplasty are advantageous because they do not require oral graft harvesting and rely on easily accessible local tissues. [12]

\section{Transplants of the oral mucosa}

Several published series have shown excellent results after urethroplasty using oral or lingual mucosa in a backbone technique [12]. While the individual series is limited by the small number of patients and the lack of long-term data, the results are indeed promising.

Oral mucosal graft uretroplasty using the ventral layer technique was presented in two separate series comprising two patients each. In the case of patients with high blood pressure, it was difficult to assess the feasibility and effectiveness of this technique because of the limited number of patients. A new modification of a ventral inlay technique using a vaginal saving approach has been described, which may minimize the risk of postoperative complications. [12]

\section{Conclusion}

Urethral stricture is an infrequent condition in women, the most dangerous etiology being stenosis of cancerous origin. Non-malignant tumoral causes such as that reported in our observation remain exceptional.

The treatment is not currently clearly codified but it is lawful to propose, for the small primitive stenosis, a minimalist treatment by dilatation or internal urethrotomy with a risk of recidivism non-negligible. In case of extensive or recurrent stenosis, it is necessary to propose a surgical treatment by plasty using pedicled flaps taken from the vagina or the labia minora or free flaps taken from the level of the oral mucosa. In case of extensive fibrous stenosis with destruction of the urethra, it is possible to propose a continous cystostomy as our patient has benefited from, an option that modifies the body's schema the least and which makes it possible to preserve continence.

\section{References}

1. Keegan KA, Nanigian DK, Stone AR (2008) Female urethral stricture disease. Curr Urol Rep 9(5):419-23

2. Pugh WS (1924) Stricture of the female urethra. Ann Surg 79:770-778.

3. Brannan D (1951) Stricture of the female urethra. J Urol;66: 242e53.

4. Smith AL, Ferlise VJ, Rovner ES (2006) Female urethral strictures:successful management with long-term clean intermittent catheterization after urethral dilatation. BJU Int;98:96e9.

5. Merimsky E (1985) Retention secondary to urethral stricture in the female. Urology;26:598.

6. Carr LK, Webster GD (1996) Bladder outlet obstruction in women. Urol Clin North Am ;23:385e91.

7. Kuo HC (2005) Videourodynamic characteristics and lower urinarytract symptoms of female bladder outlet obstruction. Urology;66:1005e9. 
8. Gutiérrez Ruiz C, Rodríguez-Escovar F, Errando Smet C, et al (2009) Female urethral stricture: etiology, diagnostic and treatment. Actas Urol Esp 33(7):794-800

9. Brannan D (1951) Stricture of the female urethra. J Urol 66(2):242-253

10. Tanello M, Frego E, Simeone C, Cosciani Cunico S (2002) Use of Pedicle Flap from the Labia Minora for the Repair of Female Urethral Strictures. Urol Int. 2002;69(2):9 -98.
11. Edwards LE, Lock R, Powell C, Jones P (1983) Postcatheterisation urethral strictures. A clinical and experimental study. Br J Urol 55(1):3-6

12. Surgical management of female urethral strictures Nathan Hoag, Justin Chee Department of Urology, Austin Hospital, University of Melbourne, Heidelberg, Victoria 3084, Australia; MURAC Health, East Melbourne, Victoria 3112, Australia 